\title{
The Construction of City Image in Short Video: A Case Study of Xi'an in the Short Video of Douyin
}

\author{
Cao Shan ${ }^{1}$ \\ ${ }^{1}$ School of I Shaanxi Polytechnic Institute, Xianyang, shannxi 712000, China \\ *Corresponding author. Email: woshuo20hao@163.com
}

\begin{abstract}
With the continuous advancement of urban modernization, the city has become an important node of global economic development and cultural exchange, and the competition among cities is inseparable from the shaping and dissemination of the city image. In recent years, Douyin short video has changed the way of media content production and dissemination with its own platform advantages, and has promoted the popularity of Xi'an, and boosted the image building of city brand rapidly. Therefore, from the perspective of "urban image communication", this paper uses content analysis, literature research and other research methods to explore the role of short video in urban image construction. Through the research of Xi'an urban image communication on Douyin short video platform, this paper discusses its strategies in content production and communication.
\end{abstract}

Keywords: City Image Construction; Douyin Short Video; Xi'an

\section{Introduction}

With the continuous development of urbanization, the competition between cities is becoming increasingly fierce, and the city image has received unprecedented attention as an intangible asset of a city. However, the image of a city is not inborn, but gradually formed in the long-term continuous exchanges and interactions with other cities and regions. Especially in the information age, the city image is not just a reappearance of city reality, but is gradually formed through the shaping of symbols and the spread. In past three years, the governments of cities such as Xi'an have fully recognized the importance of the city image, and through the use of Douyin short video App, a rapidly developing emerging medium, have helped the construction and spread of the city image and achieved remarkable results. Therefore, it is necessary for us to conduct a systematic and in-depth study on it in order to provide an effective reference for subsequent improvement and promotion.

\section{The Connotation of City Image}

\subsection{What is city image?}

According to Lewis Mumford, a famous sociologist, "city image is people's subjective impression of the city, which is formed through the combined effects of mass media, personal experience, interpersonal communication, memory and environment" [1]. In our country, the study of city image originated at the end of the last century, and Chinese scholar Li Xingguo believes that "the city image is an intuitive reflection of the city's internal quality and cultural connotation on the city's external form, which is a deep impression that the city is different from other cities" [2].

From these discussions, we can all see that the city image is not just a reappearance of city reality, but is gradually formed by various symbols and spread.

\subsection{The function of city image}

When we talk about some famous cities, we always bring them some tags. Just like perfume in Paris, Hollywood movies in Los Angeles, fashion in Milan, and in China, such as the shopping in Hong Kong, the Bund in Shanghai, Huawei in Shenzhen ... so that when someone brings perfume back from Paris, we think it must be high-end. When a Hollywood movie is released, we think it must be a big production and worth seeing. Therefore, in the eyes of ordinary people, the image of the city can be almost equal to the city's iconic industries, enterprises, or even a specific product. In the current fierce regional competition, this extended effect of " love me, love my dog" due to the city's image can enhance the sense of belonging and pride of the citizens. This has made them consciously invest in the construction and promotion of the city, and they have played a huge role in promoting the development of the tourism industry, boosting capital, attracting talents, and improving the city 's popularity and reputation. 


\section{From the Ancient Capital of the 13th Dynasty to the Net-red City-the Excavation of City Cultural Symbols in Short Videos}

In the past, when people mentioned Xi'an, they often thought of famous historical and cultural monuments and natural landscapes such as magnificent terracotta warriors and horses, and tall and steep Mount Hua. These hard cultures have made Xi'an a well-known tourist city both at home and abroad. At the same time, it has also left a heavy and vicissitudes impression to the public. On the Douyin App, Chinese Hamburger with cured meat, Xi'an Tumbler Lady Ms. Pika Chen, Soup dumplings, etc. have become the objects of everyone's pursuit, with a large number of fans. It is precisely through the integration of a large number of new elements in the short video that the public has changed the stereotype of $\mathrm{Xi}$ 'an, so that the city 's image can be displayed in an all-round three-dimensional way, thus exuding a more charming flavor.

According to the analysis of the video content related to \#Xi'an\# in the Douyin platform and the top 100 views, the content of these popular short videos mainly focuses on the following aspects:

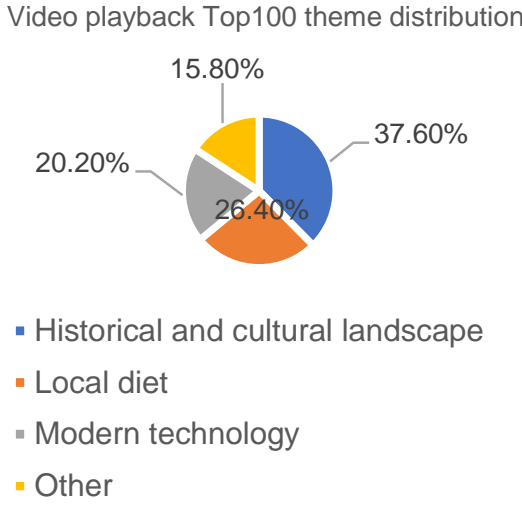

Figure 1 video playback Top100 theme distribution

\subsection{Historical and cultural landscape}

Xi'an, as the ancient capital of the thirteen dynasties, has a profound cultural accumulation, especially the 13 dynasties including Qin, Han and Tang dynasties have successively established capitals in Xi'an and created splendid cultures, making Xi'an a historical city in China and the world. In the process of building the city image, Xi'an took full advantage of this feature to build the historical and cultural landscape into the hottest content in Douyin short video. From the night view of the Bell Tower to the Xi'an City Wall, from the bronze statue of Xuan Zang at Dayan Pagoda Square to the the Tumbler Lady in Datang "never sleeps", they are all popular scenes of Xi'an's city image in vibrato. In particular, the popularity of Tumbler Lady Pika Chen is the best marketing of urban culture. Overnight, "Give me a hand" was popular all over the net, and her social appearance and dancing postures on all major social platforms including Douyin, and many immigrant tourists drove thousands of miles just to "punch cards" the smile of beauty.

\subsection{Local diet}

"People regard food as the sky". As the most profound part of Chinese culture, food and beverage occupies a pivotal position in urban development. The numerous delicacies in Xi'an have attracted the attention and lens of countless tourists. On the Douyin platform, food video shooters dig deep into the streets to discover the city's "hidden food", which makes the city image more vivid, and makes snacks that are not well-known in the past into the public's sight at the same time. The specialty snacks hidden in the streets, such as yam Ciba, meatball hula Tang, mirror cake, buckwheat noodles, cold noodles, are well known in Xi'an. The "brush pastry" has also become a popular food for users "punching", and Changan food stalls have also become a veritable "net red restaurant". The name "Sanqin set meal" gives people a familiar symbol of Cold Rice Noodles, Chinese Hamburger with cured meat and Bingfeng.

\subsection{Modern technology}

Xi'an, as an ancient capital, also has a complete modern urban civilization. Its city image propagates from the ancient capital, not just the "ancient capital". The sense of modern science and technology that is in sharp contrast to the image of Xi'an city is another object that has been talked about by countless vibrato producers. When night falls, landmark historical buildings such as Bell Tower, Dayan Pagoda, and Yongning Gate Square are lit up. In the video, the light and shadow Xi'an created by the lights are in stark contrast to the quaint city during the day, which brings infinite vitality to the night of this ancient capital, changing the city's impression of "seeing the temple in the car during the day and sleeping in the hotel at night" in one fell swoop. It achieved a beautiful scene in many places that never sleep. At the same time, the drone performance in Xi'an has become a hot topic in recent years. Thousands of drones participated in the show, highlighting the charm of Xi'an's hard-tech capital, which attracts visitors. In addition, there is a particularly popular "Song of Xi'an", with a total of more than 2 billion views, which has become a necessary background music for various videos related to Xi'an and the object of everyone's 
cover. This way of combining popular fashion elements with classic traditional culture has also brought more positive reviews to Xi'an.

These cultural symbols are like the capillaries of the city, permeating all aspects of the city, forming a more distinctive, more recognizable and three-dimensional city image.

\section{Possibility of Short Video to Promote the City Image}

\subsection{Integration of production and communication}

In the history of human communication, it has experienced many historical stages, such as oral communication, written communication, printed communication and digital communication. In this long process of development, the development of technology is undoubtedly the most important reason for promoting the transformation of communication methods. With the emergence of 5G technology, mobile short video may bring new possibilities for information dissemination.

As a UGC (user generated content) product, Douyin is low in operation threshold and users has a strong sense of participation. They are both content browsers and producers and communicators. At the same time, video has more advantages than words, pictures, audio in content communication, and the content of communication is also more three-dimensional and intuitive. After the user's independent shooting, he can use the special effects software that comes with the platform to upload it through simple editing, and easily realize the production and distribution of the content.

\section{2. "Light" interactive reading experience with vertical screen sliding}

In modern society, visual culture has become one of the most important channels for obtaining information in social life. The consumer theorist Willis once pointed out: "In a developed consumer society, consumer behavior does not need to involve economic exchange, we consume with our own eyes." ${ }^{[3]}$ The Douyin App is low on user's usage scenario, and only requires a simple hand sliding action to allow the content to be continuously played. This "light" interaction method undoubtedly increases the user's immersive experience during the viewing process, and enhances the stickiness between the user and the platform.

At the same time, with the upgrading of mobile terminals, mobile phones mostly adopt the vertical full-screen design, and people are used to using the mobile phones vertically while traditional videos are still mostly viewed on the horizontal screen, which does not meet people's reading habits. Most of the short videos in Douyin are presented in a vertical screen. Although there are certain restrictions on the length of the display screen, the details of the transmission content are enlarged, which can immerse the user in an "unconscious" during use. It also makes the use value and added value of the product fully reflected.

\subsection{Big data estimates audience preferences}

When pushing videos, Douyin App uses the powerful algorithm logic behind it to collect the data of the users' preferences when watching them, and push the video content of interest to the users. Meanwhile, it also adds the PGC videos and cooperative advertising resources that they want to promote. This kind of information push based on the user's big data collection not only conforms to the user's preferences, but also promotes the content they want to publicize. For users, it is never possible to know what the next video content is. The unpredictable content pushed by this blind selection will also stimulate the secretion of dopamine and keep people excited, so they are willing to continue to immerse themselves in subjective initiative. Even if you have content that you are not interested in, just swipe up and down without taking up too much time.

\section{Strategy of Douyin Short Video in the Construction of City Image}

\subsection{Agenda setting on the topic of "city"}

After the relevant subject initiates the challenge through the city topic in Douyin APP, the user spontaneously participates in the creation of short video content on the city topic and independently performs activities such as likes, comments, and forwarding. These behaviors can have a direct impact on all aspects of related city communication construct effects. The data found that as of March 25, 2020, there were a total of 564,000 videos on the topic of \# Xi'an \# in Douyin, with 15.53 billion views. The second place is the \# follow the Douyin play Xi'an \# topic launched by the official Douyin. There are 90,000 videos on this topic, which are played over 1.72 billion times. It can be seen that this kind of challenge topic all has a great attention.

\subsection{Use the wisdom of netizens to participate}

In the past, the discourse system of urban image construction was mainly constructed by the 
government through various traditional media, such as news reports, broadcast of urban image promotional films and other forms. In the process of carrying out these publicity activities, the whole city is often the core text, and the details related to the city image are not dealt with enough. In the Douyin platform, each user is given the ability to produce and disseminate short video texts, and city image construction has also broken through the previous official discourse system. The masses of people are no longer simply recipients of information, but have become the nodes of city image dissemination. Everyone can create and disseminate short video texts about city images from their own perspectives. In this situation, as the content producers and communicators, the public displays a richer and more solid city image, and the angle of the city image perceived by users is more diverse and grounded.

\section{Conclusion}

With its own platform advantages, Douyin Short Video has transformed the production and dissemination of media content, brought new opportunities for the construction and dissemination of city images, and brought a new look to the city image. In terms of city image communicators, the Douyin short video breaks through the previous macro-narrative model of government propaganda, greatly enhances the general public's right to speak, and enhances the public's enthusiasm for participating in city image communication. At the same time, in terms of the content of city image communication, the historical and cultural landscape, local diet, modern technology and other elements in the Douyin short video have been used as symbol carriers to change people's stereotypes of the ancient city and build a more vivid city image. Therefore, by analyzing why the Douyin App can play a role in the construction of Xi'an city image and its symbolic construction in image building, we can affirm the contribution of mobile short video platform represented by Douyin as the contribution to the construction and dissemination of city image, and provide a new idea for local governments to construct and propagate the city image, and enrich the theoretical discussion of the construction and dissemination of city image in a new era.

\section{REFERENCES}

[1] Lewis Mumford.The City in History: Its Origins, Its Transformations, and Its Prospect[J]. American Journal of Sociology, 1961(2):5

[2] Li Xingguo. Beijing Image [M]. Beijing: China
International Broadcasting Press, 2008 edition, pages $2-3$

[3] Lu Yang. Introduction to Cultural Studies [M]. Shanghai: Fudan University Press, 2008 [4] [US] Kevin. Lynch. The impression of the city [M]. Translated by Xiang Bingren. Beijing: China Building Industry Press, 1990

[4] Terence. Hobbs. Structuralism and semiotics [M]. Translated by $\mathrm{Qu}$ Tiepeng. Shanghai: Shanghai Translation Press, 1987

[5] Su Yonghua. Theory and practice of urban image communication [M]. Hangzhou: Zhejiang University Press, 2013

[6] Wang Yuwei, Mi Jiemin. Urban image communication from the perspective of semiotics: Taking Kaifeng, Henan as an example [M]. Guangzhou: Jinan University Press, 2015

[7] Ma Yena. Research on Mobile Short Video from the Perspective of Use and Satisfaction: Taking Douyin App as an Example [J]. Technology Communication. 2018.07

[8] $\mathrm{Hu}$ Xiaoyun. Analysis of the definition of city brand $[\mathrm{J}]$. Advertising concept. Theoretical Edition, December 2008.12

[9] Xu Jingyi. Douyin Short Video and Xi'an Tourism Wonder [J]. Journalism and Communication. 2018.12

[10] Wang Xiaoxin. Analysis of the content of Douyin short video in the new media environment $[\mathrm{J}]$. New Media Research, 2018.07

[11] Zhou Min, Yan Jiaqi. From "Watching" to "Participation": A Study of the Significance of Incorporated Practices in the Construction of City Images from the Perspective of New Media $[\mathrm{J}]$. Audiovisual, 2017.09

[12] D.W.F. van Krevelen and R. Poelman. A Su 